\title{
Les Cobas (Comités de Base) en Italie au cours des années quatre-vingt : naissance d'un phénomène
}

I Cobas negli anni Ottanta: nascita di un fenomeno

The CoBas: a 1980s phenomenon

\section{Rodolphe Pauvert}

\section{OpenEdition}

\section{Journals}

Édition électronique

URL : http://journals.openedition.org/cei/382

DOI : 10.4000/cei.382

ISSN : 2260-779X

\section{Éditeur}

UGA Éditions/Université Grenoble Alpes

Édition imprimée

Date de publication : 15 mars 2012

Pagination : $79-98$

ISBN : 978-2-84310-222-6

ISSN : 1770-9571

\section{Référence électronique}

Rodolphe Pauvert, « Les Cobas (Comités de Base) en Italie au cours des années quatre-vingt naissance d'un phénomène », Cahiers d'études italiennes [En ligne], 14 | 2012, mis en ligne le 15 septembre 2013, consulté le 26 mars 2021. URL : http://journals.openedition.org/cei/382 ; DOI : https://doi.org/10.4000/cei.382 


\title{
LES COBAS (COMITÉS DE BASE) EN ITALIE AU COURS DES ANNÉES QUATRE-VINGT : NAISSANCE D’UN PHÉNOMÈNE
}

\author{
Rodolphe Pauvert \\ Université de Poitiers
}

Les Cobas ou Comités de Base, sont la nouvelle forme d'expression syndicale qui voit le jour au cours des années 1980. Ils apparaissent, dans la littérature relative au syndicalisme italien, comme des objets non identifiés ou, du moins, très mal identifiés et, surtout, très peu étudiés. Bruno Fiorai, par exemple, dans son ouvrage Il sistema sindacale italiano e il principio di maggioranza, n'y fait qu'une vague allusion sans apporter la moindre explication :

[...] ainsi que les nouvelles agrégations syndicales (Cobas et formes semblables) ${ }^{\mathrm{I}}$.

Pour sa part, Sergio Turone, propose, dans son anthologie du syndicalisme italien depuis la fin de la seconde guerre mondiale, une explication peu convaincante et peu documentée :

À l'origine du phénomène a été l'introduction, dans le secteur public, d'une contractualisation à échéance triennale, semblable à celle qui est d'usage pour les salariés des entreprises privées, dont les relations salariales sont régies par des conventions collectives $^{2}$.

Afin d'essayer de définir ce que sont précisément ces Cobas et d'envisager la place qu'ils ont occupée ou qu'ils occupent encore dans le paysage syndical italien, et plus largement dans la société italienne, nous ne pouvons nous limiter à un examen factuel de la question.

I. B. Fiorai, Il sistema sindacale italiano e il principio di maggioranza, Milan, Giuffrè editore, I99I, p. 222.

2. S. Turone (dir.), Storia del sindacalismo in Italia dal 1943 al crollo del comunismo, Bari, Laterza, I992, p. $53 \mathrm{I}-535$. 
En effet, il convient, tout d'abord, de nous intéresser aux spécificités des organisations syndicales italiennes afin de comprendre le contexte qui a permis l'émergence du phénomène considéré et, surtout, afin d'essayer d'en identifier les causes et les moteurs réels.

\section{Le syndicalisme en Italie avant l'avènement des Cobas}

L'examen historique de la question syndicale en Italie ainsi que celui de l'organisation structurelle et financière des trois confédérations présentes au début des années 1980 a un double objectif. Dans un premier temps, il s'agit de mettre en évidence des cycles de vie propres à ces organismes avant de proposer une modélisation fonctionnelle du syndicalisme italien de l'époque.

\section{Contextualisation historique}

La première forme d'organisation de travailleurs qui apparait sur le territoire italique est celle des Società Operaie di Mutuo Soccorso. D'abord constituées dans le Piémont au milieu du $\mathrm{XIX}^{\mathrm{e}}$ siècle, elles jouent un double rôle : défendre les intérêts des salariés face à leurs employeurs mais également les assister, à la façon des organismes mutualistes que nous connaissons aujourd'hui ${ }^{3}$. Ainsi, par exemple, dès $1848^{4}$, une quarantaine d'ouvriers typographes turinois créent la Società dei Compositori Tipografi. Le phénomène se diffuse progressivement à d'autres secteurs et à d'autres territoires'. Dans un second temps, des fédérations vont se constituer afin de coordonner l'action des différentes sociétés de travailleurs. La première, l'Associazione fra gli operai tipografi italiani, voit le jour à Rome en $1872^{6}$.

Bien que le développement industriel de l'Italie ait quelque retard par rapport à celui de la Grande-Bretagne, l'Allemagne ou encore la France ${ }^{7}$, la classe ouvrière italienne présente à la fin du XIX ${ }^{e}$ siècle participe, à Londres, à la création de l'Association internationale ouvrière (28 septembre I864) ${ }^{8}$.

3. Sur la question des « società di mutuo soccorso » voir, par exemple, I. Barbadoro, Il sindacato in Italia, Milan, Teti, I979.

4. Année où Marx et Engels rédigent le Manifeste du parti communiste.

5. C. Perna, Breve storia del sindacato. Dalle società di mutuo soccorso al Patto federativo, Bari, de Donato, I978, p. 24-28.

6. M. Antonioli, Il sindacalismo italiano dalle origini al fascismo, Pise, Biblioteca Franco Serantini, I997, p. 15 -I6.

7. Sur la question du développement tardif de l'industrie en Italie et le subséquent développement des syndicats, voir, par exemple, S. Zaninelli, Le lotte nelle fabbriche I86I-I92I, Milan, Celuc, 1973 et G. Couffignal, Les syndicats italiens et la politique, Grenoble, Presses universitaires de Grenoble, 1978, p. 30-33.

8. C. Perna, Breve storia del sindacato, ouvr. cité, p. 29. 
Alors que le nord s'industrialise, la figure de l'ouvrier devient plus nette et les intérêts de la masse prolétaire deviennent communs. Une conscience politique se structure. En I882, elle conduit à la naissance du parti ouvrier italien, qui fonctionne davantage comme syndicat que comme parti politique $^{9}$, ainsi qu'à celle du parti socialiste en $1892^{10}$. Pour ce dernier, les Camere del Lavoro qui sont en train de voir le jour sur le territoire, représentent des structures sur lesquelles s'appuyer pour organiser la lutte

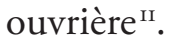

En I89I, face à la montée du socialisme et du communisme, auxquels l'Église est hostile, le pape Léon XIII invite les croyants à créer leurs propres organisations ${ }^{12}$. Le syndicalisme "blanc ", c'est-à-dire catholique se développe ${ }^{13}$.

En I900, le syndicalisme italien montre tout son pouvoir : la grève organisée le in décembre de cette année pour protester contre la dissolution de la Camera del Lavoro de Gênes conduit à la chute du gouvernement Sarocco ${ }^{\mathrm{I}}$. Les syndicalistes comprennent vite qu'en regroupant leurs forces et en coordonnant leurs actions, ils peuvent être entendus. En 1906 (29 septembre-I ${ }^{\mathrm{er}}$ octobre), à l'initiative de la Federazione Italiana Operai Metallurgici (FIOM), la Confederazione Generale del Lavoro (CGL) est constituée $e^{\mathrm{Is}}$ afin d'aller plus loin dans l'action. La convergence conduit, notamment, à la centralisation des décisions.

9. Le parti ouvrier italien n'a été présent qu’en Lombardie. Au sujet du parti ouvrier italien, voir, par exemple, ses revendications de 1888 : D. Marucco, I sindacati nella società contemporanea, Turin, Loescher editore, p. IO8-III.

IO. Au sujet de la naissance du parti socialiste italien, d'abord dénommé parti des travailleurs italien, voir C. Perna, Breve storia del sindacato, ouvr. cité, p. 5I-52.

II. La première Camera del Lavoro est celle de Milan (I89I). Elles sont formées sur le modèle des Bourses du Travail qui ont vu le jour en France en 1887 afin d'aider les travailleurs et de servir de lien entre l'offre et la demande de travail. Sur la question, voir, par exemple, C. Perna, Breve storia del sindacato, ouvr. cité, p. I2I-I22 et I. Milanese (dir.), Le camere del lavoro italiane. Esperienze storiche a confronto, Ravenne, Longo, 200I, p. I59-I90.

I2. L'encyclique Rerum Novarum est, à ce sujet, déterminante car c'est la première fois que l'Église s'exprime sur les relations dans le travail entre salariés et patronat. D. Marucco, I sindacati nella società contemporanea, ouvr. cité, p. II8-I2I. Sur la question, du lien entre religion, politique et syndicalisme, voir également F. Traniello, Dalla prima Democrazia Cristiana al sindacalismo bianco, Rome, Edizioni Cinque Lune, 1983.

13. En 1918, les forces syndicales catholiques s'uniront au sein de la Confederazione dei Lavoratori Italiani (C.I.L.) (A. Pepe, Il sindacato nell'Italia del 'goo, Messine, Rubbettino, 1996, p. 45).

I4. "Sciopero generale nel Porto e negli stabilimenti per lo scioglimento della Camera del Lavoro ", Corriere della Sera, 2I-22 décembre $1900:$ "La gravità del fatto consiste [...] soprattutto nella novità del contrasto. Non si tratta qui di operai i quali si trovino in conflitto con dei privati, pretendendo un aumento di mercede o concessioni di altra natura: bensi di operai che pongono per condizione della ripresa del lavoro la revoca di una decisione dell'autorità. " Sur la question, voir également C. Perna, Breve storia del sindacato, ouvr. cité, p. 67.

I5. Dès 1906, plus de 700 organisations syndicales l'intègrent. Sur la question, voir notamment L. Marchetti (dir.), La Confederazione Generale del Lavoro negli atti, nei documenti, nei congressi 1906-1926, Avanti!, Milan, 1962; A. Pepe, Storia della CGL I905-I9II, Laterza, Bari, I972; A. Pepe, La CGL e l'età liberale, Rome, Ediesse, 1997 . 
En I9I2, un nouveau coup est porté à la CGL : les syndicalistes révolutionnaires l'abandonnent et ils se regroupent au sein de l'Unione Sindacale Italiana ${ }^{16}$.

$\mathrm{Au}$ sortir de la première guerre mondiale, plusieurs partis politiques sont créés : le parti fasciste, le parti communiste et le parti populaire italien. Le paysage politique se construit encore un peu plus. Parallèlement, le mécontentement populaire grandit. La guerre est finie mais la crise économique sévit. Au cours du biennio rosso (1919-1920), le nombre d'inscrits à la CGL double, pour atteindre 2320 I63 et alors que le chiffre n'était que de 249039 en $1918^{17}$. Le poids des syndicats ne cesse d'augmenter, notamment grâce à la création des consigli di fabbrica ${ }^{18}$ qui permettent aux ouvriers de s'exprimer.

Face au poids qu'acquiert la masse prolétaire, la bourgeoisie décide de soutenir le fascisme pour endiguer l'avancée des mouvements ouvriers ${ }^{19}$. Les résultats ne se font pas attendre. Le début du ventennio fascista marque un coup d'arrêt brutal au débat d'idées qui a vu le jour lors de la période précédente et au ré-équilibrage qui commençait à s'opérer entre salariés et patrons ${ }^{20}$. Suite au Patto di Palazzo Chigi (1925) ${ }^{2 \mathrm{I}}$ et au Patto di Palazzo Vidoni (2 ottobre 1925$)^{22}$ signés entre la Confindustria et le régime fasciste, seul le syndicat fasciste est officiellement reconnu ${ }^{23}$. Par la suite, les syndicats sont purement et simplement dissous ${ }^{24}$.

Le syndicalisme libre n'est pas mort pour autant. Il se réorganise dans la clandestinité et il montrera toute sa force à l'occasion des grèves organisées

I6. L. Marchetti, La Confederazione Generale del Lavoro negli atti, ouvr. cité, p. I8-I9.

17. C. Perna, Breve storia del sindacato, ouvr. cité, p. 45.

18. Le premier à voir le jour est celui de la Fiat en septembre 1919. Les représentants des consigli di fabbrica sont élus par tous les salariés et non seulement par les syndiqués. Sur la question, voir par exemple F. Loreto, Il sindacalismo confederale nei due bienni rossi del Novecento I9I9-20 e I968-69, Rome, Ediesse, 2006, p. I6I-I78; G. Maione, Il biennio rosso. Autonomia e spontaneità operaia nel I9r9-I929, Bologne, Il Mulino, I975.

19. C. Perna, Breve storia del sindacato, ouvr. cité, p. I40.

20. La loi $n^{\circ} 563$ du 13 avril 1926 , «Disciplina giuridica dei rapporti collettivi del lavoro ", dite Loi « Rocco » conduit ensuite à la suppression de toutes les libertés syndicales.

2I. Il s'agit d'un accord conclu entre le patronat et le gouvernement fasciste afin que la Confindustria et que les corporations travaillent en plus étroite collaboration. Sur la question, voir, par exemple, F. Cordova, Le origini dei sindacati fascisti 1918-1926, Firenze, La Nuova Italia, Scandicci, I990, p. I79.

22. Selon l'accord, la Confindustria et la Confédération des Corporations se reconnaissent réciproquement comme représentants exclusifs des employeurs et des salariés et elles s'engagent à négocier directement les conventions collectives, abolissant ainsi les commissions internes (ibid., p. 430-43I).

23. Les syndicats « reconnus » représentent automatiquement tous les salariés des professions concernées. Les autres syndicats existent toujours mais ce ne sont plus que des associations de fait dépourvues de tout pouvoir (ibid., p. 434-442).

24. Il faut attendre le DLL n ${ }^{\circ} 369$ du 23 novembre 1944, "Soppressione delle organizzazioni sindacali fasciste e liquidazione dei rispettivi patrimoni " pour que les syndicats fascistes soient officiellement supprimés. 
dès 1943 dans le nord du pays ${ }^{25}$. Les syndicalistes jouent un rôle non négligeable dans la libération du pays. Le fait est d'importance car il donnera une légitimité indiscutée à leurs organisations pour toute la seconde moitié du vingtième siècle.

Le 4 juin 1944, la veille de la libération de Rome, le PCI, la DC et le PSI signent le Patto di Roma selon lequel la lutte syndicale ne peut s'organiser que dans l'unité. Du 28 janvier au I ${ }^{\text {er }}$ février 1945 s'ensuit le premier congrès de la Confederazione Generale Italiana dei Lavoratori (CGIL) : l'organisation syndicale unitaire voulue par le pacte ${ }^{26}$. La bourgeoisie n'a pas trop à craindre de la remontée des forces de gauche car, très vite, dès 1947, le parti socialiste et le parti communiste sont mis à l'écart du pouvoir ${ }^{27}$. Les syndicats n'arrêtent pas pour autant leur travail mais, alors que le paysage politique se recompose, le paysage syndical connaît également des transformations.

Le I4 juillet I948, suite à l'attentat commis contre le secrétaire général du PCI, Palmiro Togliatti, la CGIL décrète la grève générale. Pour manifester leur opposition à cette décision, le courant démocrate-chrétien ainsi qu'un groupe de républicains décident d'abandonner la CGIL ${ }^{28}$. En 1950, ils s'unissent au sein de la Confederazione Italiana dei Sindacati Lavoratori (CISL). De nombreux républicains laïcs et de socialistes ne se reconnaissent ni dans la CGIL ni dans les nouvelles agrégations syndicales. Aussi choisissent-ils, également en I950, de former leur propre organisation : l'Unione Italiana del Lavoro ${ }^{29}$.

Les différences idéologiques des trois confédérations syndicales ne les empêchent pas de se rapprocher à de nombreuses occasions pour mener front commun. Leur travail permet aux salariés de se voir reconnaître de nombreux droits. Le meilleur exemple est celui du Statuto dei Lavoratori ${ }^{30}$ qui, en 1970 instaure, de façon solennelle et pérenne, toute une série d'acquis sociaux. En juillet 1972, la convergence aboutit, non pas à une fusion,

25. C. Perna, Breve storia del sindacato, ouvr. cité, p. I6I-I79.

26. Sur la question du pacte de Rome et la consécutive naissance de la CGIL, voir, par exemple, G. Couffignal, Les syndicats italiens et la politique, ouvr. cité, p. 40-48; C. Perna, Breve storia del sindacato, ouvr. cité, p. I83-188.

27. Dès juin 1947, le PCI et le PSI sont écartés du gouvernement (Governo De Gasperi).

28. "Verso la fine dell'unità sindacale? I dirigenti della corrente cristiana demandano alla base ogni decisione ", Il Nuovo Corriere della Sera, I7 juillet 1948.

29. G. Couffignal, Les syndicats italiens et la politique, ouvr. cité, p. 54. Sur la question des scissions de la CGIL et des consécutives naissances de la CISL et de la UIL, voir, par exemple, A. Forbice, Il sindacato nel dopoguerra. Scissioni della CGIL e nascita della UIL e della CISL (I945-I953), Milan, Franco Angeli, I990.

30. Loi $\mathrm{n}^{\circ} 300 \mathrm{du} 20$ mai 1970, « Norme sulla tutela della libertà e dignità dei lavoratori, della libertà sindacale e dell'attività sindacale nei luoghi di lavoro e norme sul collocamento ». 
comme cela était prévu initialement, mais à la création d'une structure fédérale commune : la Federazione CGIL, CISL et UIL ${ }^{3 \mathrm{I}}$.

La convergence des trois organisations n'est pas sans rappeler celle qui avait déjà eu lieu au sein de la CGL. Les syndicats se fédèrent à l'aide d'un dénominateur commun idéologique. Toutefois, la tactique avait conduit à la scission des adhérents qui ne se reconnaissaient plus dans cette nouvelle entité, considérée trop à gauche et toujours moins à l'écoute des préoccupations de la base.

En septembre 1980, la Fiat décide de licencier I4 000 salariés et d'en mettre 23000 autres au chômage technique. S'ensuit l'occupation de l'établissement pendant près de 35 jours. Afin de protester contre cette situation, 40 ooo employés et cadres de la société défilent dans les rues de Turin. Une fois encore, le regroupement décisionnel s'est fait, semble-t-il, au détriment des différentes sensibilités ${ }^{32}$. Après cet échec et l'impossibilité de se remettre en question, les trois confédérations syndicales ne cessent de perdre du terrain : les salariés se reconnaissent de moins en moins en elles. La fédération unitaire CGIL CISL et UIL est dissoute en $1984^{33}$.

Ainsi s'achève un nouveau cycle de vie qui semble être celui du syndicalisme : des salariés d'une même sensibilité se regroupent sur la base de ce qui les rapproche. Différents groupes ainsi composés s'associent entre eux pour atteindre une masse critique face au patronat. La mécanique mise en place exclut progressivement les intérêts particuliers et nouveaux tout en permettant l'émergence d'une nouvelle structure centralisée qui paraît se replier progressivement sur sa fonction décisionnelle, en se détachant de sa base. Vient ensuite le moment où les forces marginales ou marginalisées se reconnaissent et se concentrent pour reprendre leur destin en mains en ayant, au préalable, rompu avec la mégastructure.

\section{Fonctionnement interne des trois confédérations syndicales}

Observons maintenant les trois confédérations de l'intérieur afin d'identifier les raisons sous-jacentes à l'existence des cycles de vie que nous venons de mettre en évidence. Selon l'article 39 de la Constitution de la

31. L. Bellardi (dir.), Sindacati e contrattazione collettiva dans Italia nel 1972-1974, Milan, Franco Angeli Editore, I978, p. I34-I39.

32. F. Bugno, "Dossier Sindacato-Fiat / Dopo la sconfitta. Guai ai vinti ", l'Espresso, 26 octobre I980;

S. Turone, Storia del sindacalismo in Italia, ouvr. cité, p. 388-39I.

33. B. Fiorai, Il sistema sindacale italiano, ouvr. cité, p. 20I-204. 
République italienne ${ }^{34}$, les syndicats doivent être enregistrés. En l'absence de textes légaux, adoptés en application dudit article 39, aucun syndicat n'y a jamais été contraint. Pour ces raisons, notamment, les syndicats italiens sont des associations de fait, ce qui leur concède une très grande liberté.

Malgré cette amplitude, les trois confédérations syndicales fonctionnent sur un modèle commun basé sur une structure que nous pourrions qualifier de " centrale " à laquelle vient s'ajouter une structure " périphérique ". L'objet obtenu est coiffé d'un organe à la fois fédérateur et con-fédérateur. La structure centrale s'articule sur deux axes : l'un vertical et l'autre horizontal. L'axe vertical est constitué des différents syndicats de branches et/ ou de secteurs d'activités. Pour ce qui est de la CGIL, par exemple, il s'agit des Fédérations de catégorie. Nous en dénombrons treize ${ }^{35}$ aujourd'hui qui vont de la fédération italienne des employés du commerce, de l'hôtellerie, de la restauration et des services (Filcams) aux syndicats des retraités italiens (SPI), en passant par la fédération des travailleurs de la connaissance (FLC). Chacun de ces syndicats a sa propre organisation calquée, de façon simplifiée, sur celle de la CGIL. L'axe horizontal résulte, quant à lui, du découpage territorial pratiquement identique à l'ensemble des fédérations, au niveau local et régional puis national. La Filcem (Fédération des travailleurs de la chimie, de l'énergie et des manufactures), par exemple, a un secrétariat national, puis une délégation dans chacune des vingt régions. En Émilie Romagne, la Filcem est ensuite présente, au niveau local, à Piacenza, Parme, Reggio Emilia, Modène, Bologne, Imola, Ferrara, Ravenne, Forli, Cesena et Rimini, avec, à chaque fois, un responsable. En multipliant ce découpage par le nombre de Fédérations de catégorie, la CGIL parvient à un maillage très serré du territoire.

Plusieurs organisations satellites indépendantes et/ou directement affiliées au syndicat viennent compléter le fonctionnement et les activités de la structure centrale, avec laquelle elles entrent en interaction. Pour ce qui est de la CGIL, nous en recensons aujourd'hui dix-sept. Il s'agit, par exemple, de agenquadri, sorte de syndicat pour cadres supérieurs; de federconsumatori (Fédération nationale des consommateurs) ou encore de la maison d'édition Ediesse et de l'INCA (Istituto Nazionale Confederale di Assistenza).

Tout en illustrant le rôle de ces organes périphériques, l'INCA représente un cas à part en raison de son fonctionnement interne qui repose,

34. Au sujet de l'art. 39, voir, par exemple, C. Livrea, I sindacati prima e dopo la costituzione, Palerme, Herbita, p. 53-62; B. Fiorai, Il sistema sindacale italiano, ouvr. cité, p. I-IO8.

35. <http://www.cgil.it/Organizzazione/Categorie.aspx>. 
notamment, sur les patronati. Au lendemain de la seconde guerre mondiale l'État italien doit se reconstruire. Il est impossible d'intervenir de manière capillaire dans l'ensemble du pays et d'apporter une aide à chacun des citoyens qui en éprouve le besoin. Seule l'Église, grâce aux paroisses, dispose d'une structure immédiatement opérationnelle qui couvre l'ensemble du territoire. En 1945, l'INCA voit le jour à l'initiative de la CGIL. L'objectif est d'offrir aux salariés une assistance sociale, y compris à ceux qui ne sont pas syndiqués et indépendamment de leurs appartenances politiques et religieuses ${ }^{36}$. Pour intervenir partout sur le territoire, des patronati, sorte de représentations locales, se créent aux quatre coins du pays, jusque dans les endroits les plus reculés. La CGIL aide ainsi l'État laïc à remplir sa mission sans céder le pas à l'Église. Les patronati sont officiellement reconnus en I947 par l'État qui en définit alors les missions et les limites ${ }^{37}$. En pratique, l'INCA fournit davantage qu'une simple assistance aux travailleurs. Les patronati de l'INCA aident les salariés à reconstituer leurs carrières, ils leur fournissent un relevé de situation et estiment leurs droits à pension; ils les assistent également en matière d'allocations familiales, de congé maternité, de maladie, d'accident du travail ou de chômage, par exemple. Les patronati de l'INCA, comme ceux des autres organisations syndicales ${ }^{38}$ remplissent une mission de service public reconnue par l'État.

L'importance des organes périphériques est à rechercher dans le lien que ceux-ci entretiennent avec leurs usagers. Contrairement ou, en plus, des autres organes-satellites, les patronati se trouvent dans les locaux même des syndicats dont ils dépendent. Le rôle de ces organismes n'est pas tant d'obtenir des financements de l'État mais de remplir une mission de service public tout en maintenant un dialogue avec les personnes syndiquées et en attirant de nouveaux prospects ${ }^{39}$. Il existe plus de Io 000 guichets uniques

36. A. Bruzzese, P. Gagliardi et G. Carchella, Le patronato INCA en Italie et dans le monde, Rome, INCA International, 2004.

37. Décret législatif $\mathrm{n}^{\circ} 804$ du 29 juillet 1947 , "Riconoscimento giuridico degli Istituti di patronato e di assistenza sociale ".

38. Il existe 25 patronati reconnus par l'État. Les deux principaux sont l'INCA-CGIL et l'INAS-CISL. À eux deux, ils assurent $20 \%$ des prestations fournies par l'ensemble. Voir P. De Saintignon et al., Le financement des syndicats : étude d'administration comparée. Le cas de l'Italie, Paris, Inspection générale des affaires sociales, 2004, p. 8. Pour plus de détails sur l'INAS, voir, par exemple, Istituto Nazionale Sociale, Patronato e sindacato: obiettivi comuni per il progresso dei lavoratori. INAS-CISL 1969-I973 (VII Congresso confederale CISL, Roma, I8-2I giugno 1973), Rome, Istituto Nazionale Sociale, juin 1973.

39. Ibid., p. 39-40: " [...] tutta l'azione del patronato degli ultimi anni, ed in particolare quella dell'ultimo quadriennio è stata infatti proprio rivolta al:

- maggiore collegamento alle lotte rivendicative sindacali scaturite dai rinnovi contrattuali del '69 e del '72 [...]

- maggiore collegamento alle strutture dell'organizzazione a tutti i livelli per una compenetrazione patronato-sindacato $[\ldots]$ 
ou patronati en Italie, mais également à l'étranger ${ }^{40}$. Plus de 8 ooo professionnels spécialisés travaillent pour ces structures. À elle seule, l'INASCISL aurait 2,5 millions de contacts par an. Concrètement, les patronati permettraient à la CIGL et à la CISL de recruter, respectivement, près de 250000 et 120000 nouveaux adhérents par an. L'INAS fournirait également près de $60 \%$ des nouveaux adhérents retraités ${ }^{4 \mathrm{I}}$.

L'organisation syndicale précédemment décrite est coiffée d'une structure nationale dont l'objectif est de prendre en considération les attentes et les besoins qui remontent depuis la base. Le centre confédéral de la CGIL, par exemple, est divisé en aires, en services thématiques qui couvrent l'ensemble des questions qui intéressent les salariés. Il en existe aujourd'hui vingt-et-un qui vont de « environnement et territoire » à " réformes institutionnelles ", en passant par " politiques actives du travail ${ }^{42}$.

Les statuts de la CGIL, nous permettent de comprendre comment le flux d'informations et de décisions circule dans la structure. En guise de préambule à la présentation de l'organisation, au niveau national, l'article 8 du statut de la CGIL ${ }^{43}$ précise que "l'organisation de la CGIL [...] doit constamment avoir comme objectif de promouvoir la plus grande participation des inscrits et des travailleurs et l'engagement le plus efficace vers l'unité syndicale ». À cette fin, le congrès de la CGIL se réunit tous les quatre ans.

Le Congrès est l'organe délibératif de l'ensemble de la confédération (art. I5) qui élit, notamment, le comité directif qui, à son tour, élit le secrétaire général ainsi que son secrétariat. Lors du Congrès sont également choisis les membres de la commission du programme et son président (art. I6). Le comité directif occupe une place centrale car, entre la tenue de chaque congrès, c'est lui qui joue le rôle d'organe délibératif : il a une fonction d'initiative en matière d'orientation de l'action syndicale générale, dans le respect des décisions adoptées en congrès, tout en assurant la coordination des multiples structures qui opèrent au sein de la confédération syndicale (art. I6). Pour remplir sa mission, il profite du travail de la Commission du Programme (art. I8).

\footnotetext{
- una più marcata partecipazione del patronato stesso nel momento in cui il sindacato partecipa più direttamente al migliore funzionamento per migliori prestazioni degli Istituti Previdenziali. "

4O. A. Bruzzese et al., Le patronato INCA en Italie et dans le monde, ouvr. cité, p. 7.

4I. P. De Saintignon et al., Le financement des syndicats, ouvr. cité, p. 8-I4.

42. <http://www.cgil.it/Organizzazione/CentroConfederale.aspx>.

43. <http://www.cgil.it/ChiSiamo/materiali/20090713_statuto_cgil.pdf>.
} 
Le secrétariat général est l'organe exécutif de la CGIL. De ce fait, il est chargé de mettre en ouvre les décisions du comité directif (art. I7).

Enfin, le Comité de Garantie (art. 2I) tranche les conflits internes à la structure, de ce fait il joue le rôle de " pouvoir judiciaire ".

L'examen de la structure organique des confédérations syndicales nous conduit invariablement à l'esquisse d'une structure pyramidale centrifuge. Elle est pyramidale, dans le sens où elle part d'une base très large qui englobe tous les adhérents mais, plus exactement, l'ensemble des travailleurs italiens. Elle est centrifuge, dans le sens où, pour traiter les attentes et les besoins de la base, le sommet regroupe toutes les questions, les préoccupations avant de les examiner et, éventuellement, de faire redescendre des mots d'ordre d'intervention. Un tel modèle ne laisse que peu, si ce n'est aucune place, à la multitude de particularismes qui, pourtant, sont parties intégrantes du tout.

\section{Le financement des syndicats}

Les trois confédérations syndicales fonctionnent grâce à des financements dont les sources, relativement identiques, sont assez facilement identifiables. En revanche, il est moins aisé d'en déterminer les montants avec précision. Comme nous l'avons indiqué, les syndicats sont des associations de fait : ils ne sont pas obligés de publier un bilan détaillé chaque année et leurs comptes ne sont pas vérifiés par l'État. Pour ces motifs, la fiabilité des chiffres fournis annuellement par les trois confédérations syndicales ne peut être absolue. Les données accessibles doivent donc être considérées comme des indicateurs de tendance, néanmoins suffisants pour appréhender la question abordée.

Pour les trois confédérations, les cotisations constituent le principal revenu : en tout, plus d'un milliard d'euros par an ${ }^{44}$. Selon les estimations, il s'agit ici de 70 à $75 \%{ }^{45}$ des ressources annuelles des syndicats, ce qui leur permet de maintenir leur indépendance à l'égard de l'État et de tout autre

44. P. De Saintignon et al., Le financement des syndicats, ouvr. cité, p. I7. Le chiffre peut être rapporté au nombre de membres de chaque confédération syndicale : près de 6 millions d'inscrits pour la CGIL (source : <http://www.cgil.it/ChiSiamo/Quanti_Siamo.aspx>); près de 4,5 millions pour la CISL (source : <http://www.

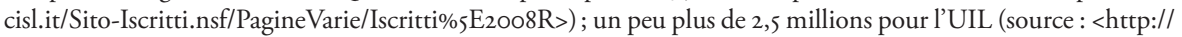
www.uil.it/organizzazione/iscritti.htm>). Les chiffres donnés sont ceux de 2008 mais ils nous permettent d'envisager la répartition globale car l'importance relative des trois syndicats, les uns par rapport aux autres, varie peu dans le temps.

45. Ibid., p. Io. 
structure privée. La spécificité italienne réside dans le fait que, jusqu’en 1995, la contribution apportée par les syndiqués est directement prélevée sur leurs bulletins de salaire ${ }^{46}$.

L'existence d'une mission de service public assurée par les syndicats, notamment par le biais des patronati, leur assure des entrées régulières. En effet, l'État italien, paie, indemnise ces derniers sur la base du nombre de dossiers traités. En contrepartie, ils sont placés sous le contrôle du ministère italien du Travail. Ladite contrepartie financière s'élève à près de 300 millions d'euros ${ }^{47}$.

Il est plus difficile, si ce n'est impossible, d'estimer le montant représenté par tous les salariés, du public et privé, qui, entièrement ou en partie, assurent des fonctions syndicales sur le lieu de travail. Les périodes ainsi occupées, prévues par la loi de $197 \mathrm{O}^{48}$, sont payées par l'employeur. Rien que pour le service public, la CGIL évalue le nombre de " détachés " à près de 2250 pour $1989^{49}$. En considérant le secteur public et privé, pour les trois confédérations, cette source de financement, presque invisible, constitue une manne non négligeable.Les autres modes de financement ne permettent pas de lever autant de fonds que ceux précédemment cités ou ils ne sont pas récurrents. Ils n'en sont pas pour autant à négliger. Lors du renouvellement d'une convention collective, par exemple, le syndicat diffuse le nouveau texte aux salariés concernés en le joignant aux bulletins de salaire que les employeurs remettent. Pour cette tâche d'information, le syndicat reçoit une indemnité forfaitaire d'environ 25 centimes d'euro par salarié syndiqué et environ 50 par salarié non syndiqué ${ }^{\circ}$. Citons également la redistribution du patrimoine financier et immobilier des syndicats fascistes, qui n'a été décidée qu'en $1977^{\text {`I }}$. Seules les organisations considérées comme représentatives au début des années 1970 ont pu bénéficier de la manne. En d'autres termes, la CGIL, la CISL et la UIL se sont vu distribuer près de $90 \%$ des biens et moyens concernés.

46. Cette mesure a été mise en place suite à la loi n ${ }^{\circ} 3 \mathrm{II}$ du 4 juin 1973 : «Estensione del servizio di riscossione dei contributi associativi tramite gli enti previdenziali. " Le référendum du II juin 1995 a ensuite conduit à son abrogation. Depuis, les modalités sont discutées puis intégrées dans les conventions collectives de branches.

47. P. De Saintignon et al., Le financement des syndicats, ouvr. cité, p. I7.

48. - Une heure par salarié et par an pour les entreprises de moins de 200 salariés; - huit heures par mois par tranche de 300 salariés et par an pour les entreprises de moins de 3 ooo salariés; - huit heures par mois par tranche de 500 salariés et par an pour les entreprises de plus de 3000 salariés.

49. P. Forcellini, « Polemiche sindacali. Tessera di Stato ", l'Espresso, 23 juillet 1989.

50. P. De Saintignon et al., Le financement des syndicats, ouvr. cité, p. I3.

5I. Loi $\mathrm{n}^{\circ} 902$ du I8 novembre 1977 : "Attribuzione dei patrimoni delle disciolte organizzazioni sindacali fasciste". 
Au vu des différentes sources de financement des trois confédérations, il est difficile de parler de rentes de situation. En effet, les cotisations des adhérents, certes importantes, sont liées à la volonté de chaque salarié de participer ou non à l'organisation. Du nombre de membres dépend également la quantité de salariés mis à disposition et l'argent perçu lors de la distribution des conventions collectives, par exemple.

Il n'en reste pas moins vrai que le modèle en place au cours des années I980 rend très difficile le développement de toute autre structure ayant pour vocation de défendre les intérêts des salariés. Tout nouveau venu dans le monde syndical se trouve alors face à deux obstacles de taille. D'une part il doit se débrouiller pour trouver les moyens d'exister matériellement car il ne peut bénéficier de l'aide de l'État, c'est-à-dire de toutes les indemnisations prévues pour " service rendu ». D'autre part, il doit trouver les moyens d'entrer en contact avec les salariés pour leur diffuser ses informations et ses propositions car il ne peut, par exemple, bénéficier des structures que constituent les patronati et/ou, tout simplement, des locaux mis à disposition dans les entreprises.

\section{L'avènement des Cobas}

Au cours des années 1980, la mécanique syndicale est, théoriquement, parfaitement organisée pour répondre aux besoins de l'ensemble des salariés, qu'ils soient syndiqués ou pas. Les règles établies permettent même aux trois grandes confédérations de maintenir une sorte de monopole dans le dialogue social. Toutefois, de nouvelles organisations font leur apparition, comme surgies ex-nihilo. Le modèle en place ne serait pas aussi parfait que voulu et il aurait fallu attendre les années 1980 pour qu'une conjoncture d'événements le mette à mal et aboutisse, de surcroît, à l'émergence de nouveaux acteurs.

\section{Un système «trop » parfait}

Selon l'article 39 de la Constitution de la République italienne, «l'organisation syndicale est libre ». D'après ces termes, il n'est fait aucun obstacle à l'émergence de nouveaux venus dans le paysage syndical. Le Statuto dei Lavoratori, reprend et détaille les dispositions prévues par la Constitution. Il est ainsi rappelé, par exemple, que les salariés peuvent librement constituer des syndicats (art. I4). Il est également souligné que les opinions syndicales sont libres (art. I) et qu'elles ne peuvent faire l'objet d'enquêtes, de la part de l'employeur, (art. 8). 
En raison de ses spécificités, l'activité salariée dans le secteur public, quant à elle, a fait l'objet d'une législation différenciée. La loi $\mathrm{n}^{\circ} 93 \mathrm{du}$ 29 mars $1983^{52}$ régit les relations syndicales dans l'administration en définissant, en limitant par conséquent, les questions qui relèvent de la négociation entre syndicats et l'employeur, c'est-à-dire l'État.

Dans la pratique, la liberté syndicale, ou plus précisément de s'organiser comme organisation de salariés en dehors des confédérations formées après-guerre, n'est pas aussi simple. En voulant codifier l'expression syndicale, le législateur a conçu des textes qui entrent en contradiction les uns avec les autres et/ou avec eux-mêmes, privant, en partie, certains dudit droit.

L'article 39 de la Constitution, par exemple, stipule que les syndicats " représentés de façon unitaire et proportionnelle au nombre d'inscrits, peuvent participer à l'élaboration de conventions collectives applicables à tous ". En d'autres termes, un groupe minoritaire de salariés dont le discours est différent et/ou nouveau, par rapport à celui des confédérations reconnues, rencontre de grandes difficultés pour mobiliser. En effet, comment recruter des membres en sachant que les représentants de l'organisation ne peuvent pas, légalement, participer à la discussion "salariatpatronat » car elle n'est pas reconnue comme " représentative », c'est-à-dire qu'elle n'a pas encore assez d'adhérents pour obtenir un siège à la table des négociations. L'obstacle s'ajoute à ceux déjà évoqués.

Pour ce qui est du Statuto dei Lavoratori, ses concepteurs ont, par exemple, instauré les Rappresentanze Sindacali Aziendali afin de permettre aux différentes sensibilités de s'exprimer sur le lieu de travail, mettant ainsi fin à toutes les pratiques antérieures. L'article 19 du Statuto dei Lavoratori indique que des Rappresentanze sindacali aziendali peuvent être constituées à l'initiative des travailleurs dans chaque unité de production ». Il est ensuite précisé que cela doit se faire "dans le cadre [...] des associations syndicales, non affiliées aux confédérations précédemment citées ${ }^{53}$, qui sont signataires de conventions collectives de travail nationales ou provinciales en vigueur dans l'unité de production ». Pris individuellement, le texte ne montre pas de limitation au droit syndical, bien au contraire, il invite chacun à participer à la discussion entre salariés et patrons.

Cependant, l'examen de l'article 39 de la Constitution nous a permis de voir que seules les organisations qui sont officiellement reconnues peuvent participer à la négociation. De ce fait, les organisations, minoritaires, se

52. Loi ${ }^{\circ} 93$ du 29 mars 1983, "Legge quadro sul pubblico impiego ».

53. Il s'agit ici des trois grandes confédérations syndicales : CGIL, CISL et UIL. 
voient également priver du droit de participer aux Rappresentanze Sindacali Aziendali. Il peut paraître compréhensible que des organisations quantitativement marginales ne participent pas à la négociation collective qui aboutira à la rédaction de règles applicables pour tous. En revanche, l'impossibilité, en pratique, de participer aux structures consacrées à l'expression apparait comme une limitation aux droits fondamentaux et surtout, comme un appauvrissement du débat d'idées.

Les longues luttes salariales ont permis d'acquérir des droits face au patronat, qu'il soit privé ou public. Pour garantir ces droits, le législateur les a codifiés afin de les "sceller " dans la République italienne.

Comme nous venons de le démontrer, nous nous retrouvons très vite confrontés aux limites du droit. D’une part, en prévoyant de façon détaillée des situations afin de prévenir tout contournement de la loi, le législateur crée des textes qui entrent en conflit, desservant ainsi la cause entendue. D'autre part, même en offrant des textes suffisamment souples pour qu'ils soient adaptés aux nouveaux contextes historiques qui se succéderont, le législateur ne peut prévoir toutes les situations. L'homme de Loi ne peut se projeter qu'à partir de ce qu'il connait. Avec le recul temporel dont nous bénéficions, il paraît difficilement envisageable que les constituants, par exemple, aient pu imaginer à quel point la société italienne, en l'occurrence, allait devenir une structure aussi complexe.

Toutefois, il ne faut pas oublier que les lois et même la loi fondamentale vivent, c'est-à-dire qu'elles évoluent, qu'elles sont modifiables. Certes, dans le cas d'espèce, les salariés minoritaires ne peuvent se satisfaire d'une telle réponse mais n'oublions pas que c'est en raison de sa trop grande souplesse, par exemple, que le Statut Albertin a permis l'arrivée au pouvoir du fascisme. Le législateur ne peut que suivre le développement de la société et difficilement le précéder.

\section{$L e(s)$ élément $(s)$ déclencheur $(s)$}

Au cours du $\mathrm{Xx}^{\mathrm{e}}$ siècle, la société italienne, à l'instar des autres nations, n'a cessé de muer. Les cycles de développement se sont faits de plus en plus rapides. L'examen du contexte propre à la société italienne de l'époque où est apparu le phénomène Cobas doit permettre de mettre en évidence le terrain favorable qui est alors en place ainsi que le(s) élément(s) déclencheur(s).

Au cours des années 1980, la société italienne est très différente de ce qu'elle était après-guerre. De société agricole, l'Italie est devenue industrielle 
avant d'entrer dans l'ère des services ${ }^{54}$. Par conséquent, la figure du salarié n'est plus une mais multiple. Les préoccupations d'un salarié d'une boutique d'informatique ne sont pas les mêmes que celles de l'ouvrier sur la chaîne de montage, par exemple. Le temps de travail se décompose désormais de nombreuses façons : travail temporaire, travail saisonnier, travail à temps partiel, etc. ${ }^{5}$. Parallèlement, le chômage ne cesse de progresser, passant de 7,6 \% en 1980 à II,I \% en $1986^{56}$.

La situation des salariés du secteur public évolue également de façon considérable. Citons simplement la loi $\mathrm{n}^{\circ} 210 \mathrm{du}$ i7 mai 1985 (Istituzione dell'ente "Ferrovie dello Stato ») qui a ouvert la porte à la transformation des chemins de fer, en changeant son statut, de Azienda autonoma en Ente delle Ferrovie dello Stato. L'État a ainsi permis le début de la mutation de cette administration en entreprise privée, mutation qui n'est pas encore achevée à ce jour.

Malgré cela, tout se passe comme si les syndicats et les pouvoirs publics ne prenaient pas la mesure du changement ${ }^{57}$. Le modèle syndical en place ne permet pas à ces voix "nouvelles " de se faire entendre comme elles le souhaiteraient. L'État ne semble pas avoir davantage conscience de la situation. La loi $\mathrm{n}^{\circ} 93 \mathrm{du} 29$ mars 1983 , par exemple, ne fait aucune distinction entre les différentes catégories de salariés qui opèrent au sein de l'école. De cette façon, le personnel administratif et les enseignants qui, pourtant, correspondent à deux réalités distinctes, sont traités de la même façon.

Comme nous l'avons démontré, les contradictions législatives ne permettent pas aux voix dissidentes ou simplement nouvelles de se faire entendre au sein de l'entreprise. Toutefois, l'article 40 de la Constitution ${ }^{58}$ qui stipule que "le droit de grève s'exerce dans le cadre des lois qui le régissent " va permettre aux nouveaux venus d'avoir voix au chapitre. En effet, au cours des années 1980, les lois organiques censées réglementer

54. Alors que les secteurs primaires et secondaires déclinent, les services occupent déjà plus de la moitié de la population active en 1982. G. Baglioni (dir.), Le relazioni industriali in Italia e in Europa negli anni 1980, Rome, Edizioni Lavoro, 1989, p. 297.

55. B. Fiorai, Il sistema sindacale italiano, ouvr. cité, p. 204-213.

56. G. Baglioni, Le relazioni industriali in Italia, ouvr. cité, p. 297-334.

57. Un signal a pourtant été envoyé aux syndicats : alors que le nombre d'inscrits aux différentes confédérations syndicales a progressé de 5,5\% entre 1977 à 1986 (respectivement 845996 inscrits et 8928 202) grâce à l'apport des retraités, le nombre d'inscrits occupant un emploi a, sur la même période, décru de $15,5 \%$, soit I II7 242 inscrits de moins. Globalement, le taux de syndicalisation chez les actifs, passe de 48,7\% en I977 à 39,7 \% en 1986 (G. Baglioni, Le relazioni industriali in Italia, ouvr. cité, p. 33I-332).

58. Au sujet de l'art. 40 et du droit de grève voir, par exemple, C. Livrea, I sindacati prima e dopo la costituzione, ouvr. cité, p. I29-I46. 
l'organisation des grèves n'ont toujours pas été adoptées ${ }^{59}$. Ainsi, ceux qui ne peuvent s'exprimer sur leurs lieux de travail peuvent, en revanche, interrompre leurs activités et descendre dans la rue à chaque fois que cela leur semble utile.

Grâce à la faille ou l'opportunité, selon les points de vue, qu'offre l'article 40 de la Constitution, les grèves ne cessent de se multiplier au milieu des années 1980. Le nombre d'heures chômées semble croître de manière exponentielle. Au cours du premier semestre 1987, près de 3,4 millions d'heures de travail sont perdues pour les seuls secteurs des transports aériens et ferroviaires ${ }^{60}$.

Les mouvements de contestation que connait alors l'Italie bénéficient d'un réel effet de surprise car leurs instigateurs sont complètement inconnus du paysage syndical. Les nouvelles organisations qui se forment spontanément pour défendre les droits des salariés, en lieu et place des syndicats officiels, sont, pendant quelque temps, qualifiés de "sindacatini $i^{61}$ ". En fait, il s'agit de coordinations de salariés qui se constituent de façon spontanée pour porter leurs voix et, le plus souvent, pour contester le contenu des conventions collectives qui ont été signées par les trois confédérations syndicales ou qui sont sur le point de l'être ${ }^{62}$.

Le phénomène, d'abord apparu dans les écoles italiennes au cours de l'année 1986, se diffuse ensuite aux chemins de fer. En 1987, et ce en moins de trois mois, $90 \%$ des 23000 cheminots des Ferrovie dello Stato adhèrent au mouvement. Ils estiment que les syndicats traditionnels ne les défendent pas, qu'ils sont rangés du côté des patrons. Les militants des coordinations spontanées demandent, 400 ooo lires d'augmentation mensuelle pour les premiers et 300 ooo lires pour les seconds $s^{63}$.

Les salariés d'autres branches, publiques ou privées, comprennent très vite qu’ils peuvent, eux aussi se « défendre », sans passer par l'intermédiaire

59. Il faudra attendre la deuxième moitié des années 1980 pour que les hommes politiques commencent à se pencher sérieusement sur la question du droit de grève (voir P. Forcellini, " Come cambia la Cgil. L'ambizione di Bruno ", l’Espresso, Is janvier 1989).

6o. T. Fazzolari, "Il morso del Cobas ", l'Espresso, I7 janvier I988.

6I. P. Forcellini, « La crisi del sindacato. Base per asprezza ", l'Espresso, 2I juin I987.

62. Les Cobas revendiquent ouvertement cette opposition aux syndicats traditionnels et à l'État, comme nous pouvons, encore aujourd'hui, le lire sur la page d'accueil du site du comité de base de l'école : "Un organismo politico-sindacale-culturale nato per rappresentare realmente gli interessi dei lavoratori della scuola, in contrapposizione alla politica dei Governi e dei sindacati confederali. " (<http://www.Cobas-scuola.it/Cobas.html>) Au sujet de la convention collective, voir, par exemple, l'étude portant sur la négociation entre acteurs sociaux entre 1972 et 1974 dans L. Bellardi, Sindacati e contrattazione collettiva dans Italia, ouvr. cité (mentionnons également que plusieurs conventions collectives sont annexées à l'ouvrage).

63. C. Mariotti, «Vita da Cobas», l'Espresso, 4 octobre 1987. 
des trois confédérations. Ils décident ainsi d'imiter le modèle mis en place par les enseignants et les cheminots : les grèves se multiplient dans tous les secteurs d'activité. Le pays est très souvent bloqué. En janvier 1988, par exemple, les avions, les bateaux et l'école sont à l'arrêt ${ }^{64}$. En avril, ce sont, entre autres, les salariés de Fiat et de Olivetti qui se mettent en grève pour protester contre le contenu des accords qui sont sur le point d'être signés par les syndicats ${ }^{65}$.

Rapidement, il est trouvé un nom aux « sindacatini ». Le terme retenu est Cobas, pour « comitati di base». Le manque de coordination entre les différents mouvements ne permet pas de bénéficier complètement du potentiel que représentent ces dizaines de milliers de militants éparpillés sur le territoire. Pour ce motif, notamment, les Cobas vont très vite chercher à s’organiser malgré la mosaïque de réalités qu’englobe ce terme générique. La nouvelle force syndicale se veut différente des modèles traditionnels.

Il est impossible de dater le moment précis où des discussions informelles conduisent quelques salariés à se regrouper pour proposer un nouveau modèle syndical. Il est néanmoins permis d'étudier la façon dont ils voient le jour et celle dont ils se développent.

Dès leur constitution en 1986, les premières coordinations spontanées de l'école se sont appelées Comitati di Base della Scuola, c'est-à-dire, Co.Ba.S. ${ }^{66}$, avant que le nom ne soit repris pour l'ensemble des phénomènes similaires. Le fait de s'attribuer un nom peut paraître anodin mais il correspond à la conscience des acteurs qu'ils viennent de mettre en place une structure. En se coordonnant, les différents comités de base de l'école ont pu mobiliser un très grand nombre d'intervenants dans l'école italienne, de façon simultanée et sur l'ensemble du territoire. En raison du succès rencontré, très vite, en $1988^{67}$, des enseignants décident de constituer la Gilda degli Insegnanti pour défendre spécifiquement leurs intérêts et non plus tous ceux des salariés de l'école, comme c'est encore le cas pour les comités de base de l'école.

De la même façon que pour l'école, différentes catégories de comités de base se sont constituées dans les chemins de fer. En raison de la multiplicité des métiers, leur nombre n'en a été que plus important. Citons,

64. T. Fazzolari, "Il morso del Cobas ", art. cité.

65. P. Forcellini, «A Mirafiori si cambia », l'Espresso, io avril 1988.

66. F. Sebastiani, «Sindacalismo di base e democrazia sindacale: dall'autunno caldo quale modello di sindacato ", Proteo, n' 2, 2002.

67. La Gilda degli insegnanti est officiellement née le 6 mars 1988 (<www.gildains.it>). 
notamment, le Coordinamento Macchinisti Uniti, le Cobas Capistazioni, le Cobas dei Manovratori, etc. ${ }^{68}$.

En voulant répondre de façon spontanée à des questions qui ne concernent qu'une catégorie de personnels, les Cobas tendent à se démultiplier. Ils sont ainsi davantage à l'écoute des salariés en s'intéressant uniquement à une question particulière mais cette tactique ne fait qu'augmenter le nombre d'intervenants qui doivent discuter ensemble pour concerter des actions plus importantes. Il est impossible de connaître le nombre de comités de base qui ont existé et qui existent encore tant ils peuvent être éphémères ou ne correspondre qu’à un micro-intérêt local.

L'apparition du phénomène étudié est due aux facteurs que nous avons précédemment identifiés, comme la possibilité de faire grève sans avoir à respecter des règles contraignantes ou à la complexification de la figure salariale au cours des années 1980. Toutefois, il faut aller plus loin dans notre recherche car ces éléments n'expliquent pas, à eux seuls, pourquoi les salariés ont presque subitement décidé d'agir indépendamment des syndicats officiels. En effet, les possibilités offertes, par les textes, de manifester existaient déjà auparavant. Après les deux chocs pétroliers, par exemple, les salariés connaissaient déjà des difficultés nouvelles et variées.

Il convient de nous intéresser aux chefs de file de ces nouvelles organisations ainsi qu'aux mobilisations qui ont été organisées pour avancer dans la compréhension du sujet.

Un article de l'Espresso du 4 octobre $1987^{69}$ nous présente des portraits de membres des Cobas. Les personnes présentées sont au nombre de quatre :

- Maria Carlo Gullotta, 36 ans, enseignante d'anglais à Rome, et ancienne adhérente de la CGIL-école qui est maintenant porteparole du Cobas de l'école;

- Fabio Protano, 37 ans, machiniste aux chemins de fer, il fait partie de la coordination nationale composée de trente "sages ";

- Roberto Todini, 39 ans, enseignant de lettres à Ostia, qui n’avait jamais été attiré par les syndicats traditionnels qui lui semblaient trop éloignés de ses préoccupations;

- Patrizia Cerquillini, 29 ans, machiniste auxiliaire à Turin, elle avait la carte de la CGIL mais elle l'a abandonnée au profit des Cobas dont elle est devenue représentante nationale.

68. <http://www.fdca.it/sindacale/ferrovie-ristrutturazione.htm>.

69. C. Mariotti, «Vita da Cobas », art. cité. 
Le tableau qui est dressé résume bien la situation. Les personnes qui choisissent d'adhérer aux comités de base sont relativement jeunes, elles sont nées après la guerre et ne reconnaissent plus les syndicats traditionnels comme des icônes auxquelles il convient de pardonner les écarts ou la lenteur d'adaptation. Rappelons que les syndicalistes ont aidé à participer à la libération de l'Italie puis à la construction de la République ainsi qu’à la reconnaissance des droits des travailleurs. La page semble tournée, tout ce qui compte est ce qui se passe dans la réalité immédiate.

Par ailleurs, les nouvelles organisations, qui peuvent être créées de toutes pièces par une poignée de salariés, permettent à chacun d'obtenir rapidement une place à un rang élevé s'il le souhaite. Tous ces nouveaux acteurs ont ainsi le sentiment d'être les maîtres du changement, changement qu'ils choisissent et qu'ils ne subissent plus : de la part des patrons ou des mégastructures syndicales.

L'autre élément qui explique le développement du mouvement engagé est la rapidité des résultats obtenus. En effet, si les premières actions engagées ne permettaient pas de changer les décisions prises, il faudrait davantage de temps pour convaincre les autres salariés de se lancer dans des organisations qui n'ont aucune existence officielle aux yeux des représentants des différents partenaires sociaux. Rappelons les nombreuses grèves organisées par les comités de base des enseignants au cours des années I986 et $1987^{70}$ qui parviennent à obliger l'État à prendre en compte une partie de leurs demandes ou encore la grève des cheminots du 28 juillet 1987 qui paralyse $60 \%$ du trafic ${ }^{71}$. Le résultat le plus spectaculaire est obtenu par les salariés de Fiumicino en 1988. Alors que les 195 représentants syndicaux (CGIL-CISL-UIL) viennent juste de signer la nouvelle convention collective au nom des douze mille salariés de l'établissement, une coordination née spontanément parvient à mobiliser $75 \%$ du personnel pour que l'accord soit revu ${ }^{72}$. La victoire est fracassante, les comités de base sont reconnus de fait alors que la place des syndicats traditionnels est, une fois encore, remise en question.

\footnotetext{
70. Ibid.

7I. "I convogli soppressi sono stati il 60 per cento. [...] A Torino hanno incrociato le braccia quasi il novanta per cento dei macchinisti, a Verona l'si per cento [...]. Ci sono anche quote più basse, che però non scendono al di sotto del 40\% (a Reggio Calabria ci si aggira intorno al 42 per cento). " (M. Cianca, "Lo sciopero dei macchinisti Cobas ha messo in ginocchio tuti i principali collegamenti. La disfatta delle ferrovie ", Corriere della Sera, 28 juillet 1987.)

72. G. Lerner, « Nell'hangar dei ribelli », l'Espresso, I7 avril 1988.
} 
L'examen synchronique du phénomène syndical nous a permis de démontrer que la naissance des Cobas au milieu des années 1980 marque le début d'un nouveau cycle de vie de la représentation salariale. Invariablement, les structures représentatives des travailleurs semblent converger pour se transformer en mégastructures qui se détachent progressivement de la base. Arrive ensuite le moment où quelques individus viennent remettre en question cet ordre établi et créent de nouvelles entités.

D'un point de vue diachronique, l'émergence des Cobas a lieu à un moment où la société italienne accélère sa mutation. La génération en place n'est plus celle de l'après-guerre qui reconnaît le rôle des syndicats dans la reconquête de la liberté, c'est désormais celle d'une jeunesse plus individualiste qui veut améliorer ses conditions de vie et, en particulier, de travail sans plus attendre. La force de frappe est si spontanée et virulente que le phénomène Cobas est presque perçu comme une sorte de «bing bang " dans le panorama syndical italien qui, lui, est très structuré, verrouillé et long à mobiliser.

Face à cette évolution, les confédérations syndicales n'attendent pas pour réagir. Dès 1987, elles cherchent à combler la brèche qui a permis le " débordement ". De concert, elles demandent l'adoption d'une loi qui vienne régir le droit de grève, en particulier dans les services publics ${ }^{73}$ afin de priver les Cobas de leur unique moyen de mobilisation. Parallèlement, l'électrochoc conduit les trois confédérations à se rapprocher de la base et à se relancer dans la lutte syndicale sur le terrain ${ }^{74}$. Il est même question de reconstituer un front commun, comme lors de la lutte contre le nazifascisme ou lors de la lutte pour les droits des travailleurs au tournant de la décennie $1960-1970^{75}$.

La guerre syndicale n'aura pas lieu. De leur côté, en se structurant, en convergeant, les Cobas entrent progressivement dans le cycle de vie syndical précédemment décrit. Eux aussi ne résistent pas à la force centrifuge qui semble inhérente aux organisations italiennes représentatives des salariés : l'atteste, par exemple, l'existence de l'actuelle Confederazione dei Comitati di $\mathrm{Base}^{76}$.

73. Sur la question, voir, par exemple, P. Forcellini, "Sciopero in Gabbia. Le polemiche nel sindacato ", l'Espresso, 25 octobre 1987; P. Forcellini, " Il morso del Cobas. Scioperi selvaggi », l'Espresso, 6 décembre 1987.

74. P. Forcellini, "Il morso del Cobas», art. cité et Id., "Come cambia la CGIL. L'ambizione di Bruno ", art. cité.

75. G. Lerner, "Affacciarsi al Duemila con un'organizzazione unica ", l'Espresso, 29 avril 1990.

76. <www.Cobas.it>. 